\title{
A PRODUÇÃO CIENTÍFICA NA GRADUAÇÃO EM ENFERMAGEM (1997 A 2004): ANÁLISE CRÍTICA
}

\author{
UNDERGRADUATE SCIENTIFIC PRODUCTION IN NURSING (1997 TO 2004): A CRITICAL ANALYSIS \\ PRODUCCIÓN CIENTÍFICA EN CURSOS DE PREGRADO EN ENFERMERÍA (1997 A 2004): ANÁLISIS \\ CRÍTICO
}

\section{Kelly Graziani Giacchero ${ }^{1}$, Adriana Inocenti Miasso ${ }^{2}$}

RESUMO: Este estudo tem o propósito de identificar o perfil da produção científica da graduação em enfermagem apresentada no Simpósio de Iniciação Científica da USP (SIICUSP) entre os anos de 1997 a 2004, descrever fatores que podem influenciar o perfil dessa produção e estabelecer uma comparação com o estudo de PEREIRA et al (1999) que realizou estudo no período de 1993 a 1996 do referido evento. A metodologia baseou-se na análise dos resumos referentes à área de enfermagem nos anais do SIICUSP do período citado mediante a classificação por tipos de pesquisa, temáticas abordadas, agências fomentadoras e instituições/departamentos de origem. Identificou-se que a maioria dos estudos é financiada por agências de fomento, houve predomínio de pesquisas não-experimentais e os aspectos sócio-culturais foram destaque entre as temáticas abordadas. As instituições públicas são as que mais contribuíram na produção de conhecimento científico. Tais características são similares às encontradas por PEREIRA et al (1999) no período de 1993 a 1996 e a análise comparativa dos achados da produção entre 1997 a 2004 revelou, ainda, um aumento progressivo do número de trabalhos apresentados, bem como a crescente participação de outras universidades no SIICUSP (que até 1996 era restrito à USP). Concluiu-se que a iniciação científica é importante etapa da formação do pesquisador e espaço para o desenvolvimento da cultura de consumo e produção do conhecimento. É fundamental ainda a observação de que ela não se desvincule das necessidades da sociedade atual e dos assuntos que motivam o aluno, visando garantir o crescimento, a qualidade e a valorização da produção de conhecimento na área da enfermagem.

PALAVRAS CHAVE: Pesquisa, Estudantes de enfermagem, Enfermagem.

ABSTRACT: This study aims to identify the profile of undergraduate scientific production in nursing presented at the University of São Paulo Scientific Initiation Symposia (SIICUSP) between 1997 and 2004 , to describe factors that can influence the profile of this production and to make a comparison with the study by PEREIRA et al (1999), which studied the same event between 1993 and 1996. The methodology was based on the analysis of nursing abstracts in SIICUSP proceedings during the studied period, which were classified according to research types, addressed themes, funding agencies and institutions/departments of origin. We identified that most studies were funded by agencies, that nonexperimental research predominated and that sociocultural aspects stood out among the research themes. Public institutions provided the greatest contribution to scientific knowledge production. These characteristics are similar to those found by Pereira et al (1999) between 1993 and 1996. The comparative analysis between the production from 1997 and 2004 and PEREIRA et al's results (1999) also revealed a progressive rise in the number of presented studies, as well as the increasing participation of other colleges in SIICUSP (restricted to USP until 1996). Scientific initiation is an important step in research training and a space to develop a knowledge consumption and production culture. Attention is fundamental to avoid scientific initiation from moving away from current society's needs and from the subjects that motivate students, with a view to guaranteeing the growth, quality and valuation of nursing knowledge production.

KEY WORDS: Research, Students Nursing, Nursing.

RESUMEN: La finalidad de este estudio es identificar el perfil de la producción científica de pregrado en enfermería presentada en el Simposio de Iniciación Científica de la Universidad de São Paulo, Brasil (SIICUSP) entre 1997 y 2004, describir factores que puedan influenciar el perfil de esa producción y establecer una comparación con el estudio de PEREIRA et al (1999), que estudió el referido evento entre 1993 y 1996. La metodología se basó en el análisis de los resúmenes referentes al área de enfermería publicados en los anales del SIICUSP del período citado, mediante la clasificación por tipos de investigación, temáticas tratadas, agencias fomentadoras e instituciones/departamentos de origen. Identificamos que la mayoría de los estudios es financiada por agencias, que predominaron las investigaciones no-experimentales y que los aspectos socioculturales se destacaron entre las temáticas tratadas. Las instituciones públicas fueron las que más contribuyeron en la producción de conocimiento

\footnotetext{
${ }^{1}$ Aluna do terceiro ano de graduação em Enfermagem da Escola de Enfermagem de Ribeirão Preto - Universidade de São Paulo. Bolsista do Programa Especial de Treinamento (PET). Ribeirão Preto - SP. E-mail: kellygiacchero@yahoo.com.br

2 Enfermeira. Professora Doutora do Departamento de Enfermagem Psiquiátrica e Ciências Humanas da Escola de Enfermagem de Ribeirão Preto - Universidade de São Paulo. Ribeirão Preto - SP E-mail: amiasso@eerp.usp.br
} 
científico. Tales características son similares a aquellas encontradas por PEREIRA et al (1999) en el período de 1993 a 1996. El análisis comparativo de la producción de 1997 a 2004 con los resultados de PEREIRA et al (1999) reveló todavía un aumento progresivo en el número de trabajos presentados, además de la creciente participación de otras universidades en el SIICUSP (que hasta 1996 era restricto a la USP). Se concluye que la iniciación científica es una etapa importante de la formación del investigador y un espacio para el desarrollo de la

\section{INTRODUÇÃO}

$\mathrm{Na}$ área da saúde, constitui-se uma preocupação crescente dos diversos profissionais o aprimoramento de conhecimentos técnicos e científicos visando qualificar cada vez mais o nível de assistência prestada ao cliente, família e comunidade (SOUSA \& BARROS, 1998). Nesse contexto, encontra-se o enfermeiro que tem em suas mãos algo precioso que deve ser desenvolvido em todos os momentos: o cuidado com outro. Cuidado este que envolve todo um aparato de informações, um conhecimento que não é algo acabado, mas uma construção que se faz e refaz de forma dinâmica (CAMACHO \& SANTO, 2001). A atividade de pesquisa, por exemplo, se constitui estratégia fundamental para a construção desse conhecimento.

As teorias e o conhecimento gerados a partir de pesquisa em enfermagem são essenciais para o estabelecimento de uma base científica que garanta a qualidade do cuidado e a credibilidade profissional (MENDES, 1991).

Assim, a atividade de pesquisa é um dos compromissos sociais da enfermagem. A produção científica em enfermagem no Brasil intensificou-se e passou a buscar embasamento teórico metodológico a partir da década de 70, fruto da reforma Universitária de 1968 (COLLET et al, 2000). A pesquisa pode e deve ser estimulada desde a graduação, por meio da iniciação científica.

A pesquisa é fundamental no processo de educação, é uma maneira acadêmica própria de educar, pois incita um questionamento que é reconstruído constantemente (DEMO, 1996). Entretanto algumas vezes, a educação profissional em nossa sociedade coíbe a criatividade e a individualidade dos alunos, por oferecer-lhes, como alternativa formal, a aquisição passiva de conhecimentos, pois o processo de formação e capacitação de recursos humanos necessita estar ligado ao desenvolvimento da criticidade do aluno para a habilitação de um profissional ativo e capaz de articular seus pensamentos e idéias (BAGGIO, 2006).

A iniciação científica permite que o aluno tenha noções teóricas e metodológicas de pesquisa, busca incentivar-Ihe o espírito questionador, amplia seus conhecimentos sobre os assuntos pesquisados, enriquece a sua formação escolar e fornece-lhe o cultura de consumo y producción del conocimiento. Es fundamental también atentar para que ella no se desvincule de las necesidades de la sociedad actual y de los asuntos que motivan el alumno, con objeto de garantizar el crecimiento, la cualidad y la valorización de la producción de conocimiento en el área de la enfermería.

PALABRAS CLAVE: Investigación, Estudiantes de Enfermería, Enfermería.

alicerce para a continuidade em programas de pósgraduação, principalmente se a opção feita for a carreira acadêmica (PEREIRA et al, 1999).

Deste modo, assegura-se o compromisso de formar enfermeiras(os) cidadãs(ãos), capazes de pensar e agir criticamente frente às demandas coletivas, reduzindo a alienação acadêmica e promovendo um processo de formação mais próximo das necessidades de desenvolvimento. Com isso, proporciona-se à sociedade uma força de trabalho compatível com as necessidades de interpretação e transformação da realidade (FERNANDES et al, 2000).

O contexto histórico em que a pesquisa é realizada está profundamente articulado com as tendências da mesma, uma vez que, ao mesmo tempo em que a enfermagem é condicionada pelo meio onde atua, ela, enquanto prática social, também exerce influência na sociedade.

Assim, o docente de enfermagem é capaz de criar pontes entre a graduação e a pós-graduação Stricto Sensu ao catalisar ambos os níveis do processo educacional. Deste modo, os graduandos são estimulados à atividade de pesquisa e o perfil da pesquisa da graduação recebe influencia direta daquela desenvolvida na pós-graduação (NASCIMENTO et al, 2002).

Com a valorização da iniciação científica entre os graduandos e sua expansão nas universidades, é necessário que os alunos possam expor seus trabalhos e passem por um processo de avaliação de sua pesquisa, não apenas para eventuais correções, mas, principalmente, para medir a abrangência dos seus efeitos. Os Simpósios de Iniciação Científica são importantes para atender a essas necessidades. O Simpósio Internacional de Iniciação Científica da Universidade de São Paulo (SIICUSP) teve início em 1993 com 403 participantes e no ano de 2003 atingiu a marca de 4000 resumos, com o envolvimento de mais de 13000 autores. Atualmente, o evento se constitui na maior mobilização acadêmica do gênero no país (SIICUSP, 2000; SIICUSP, 2003), por isso foi escolhido como fonte de coleta de dados para caracterizar a pesquisa na graduação em enfermagem.

Este trabalho teve como propósito analisar o perfil da produção científica da graduação em enfermagem apresentada no Simpósio de Iniciação 
Científica da USP (SIICUSP) entre os anos de 1997 a 2004, descrever fatores que podem influenciar o perfil dessa produção e estabelecer uma comparação com o estudo de PEREIRA et al (1999) que realizou estudo no período de 1993 a 1996 do referido evento.

Todo recorte feito para analisar o que se tem pesquisado apresenta limitações, pois, qualificar e quantificar a produção científica em nossa área é uma tarefa extremamente complexa, tendo em vista a diversidade dos aspectos que devem ser considerados (GUTIÉRREZ et al, 2002). Entretanto, por essa análise realizada será possível identificar quais aspectos temáticos têm permeado a pesquisa em enfermagem, suas tendências e áreas que ainda necessitam ser exploradas.

\section{METODOLOGIA}

Trata-se de um estudo bibliográfico com abordagem quantitativa. Para realização da investigação, houve um preparo mediante a leitura de artigos relacionados à metodologia científica, à graduação em enfermagem, sobre pesquisa na área de enfermagem bem como, de documentos referentes às agências de fomento.

Foram utilizados como fonte de coleta de dados, os resumos contidos na área da enfermagem dos Anais do Simpósio Internacional de Iniciação Científica da Universidade de São Paulo (SIICUSP), nos anos de 1997 a 2004, conforme apresentado no Quadro 1.

Quadro 1: Material bibliográfico utilizado para coleta dos dados.

\begin{tabular}{|c|c|c|c|c|}
\hline $\begin{array}{c}\text { Evento } \\
\text { (Programa e resumos) }\end{array}$ & Ano & Volume & $\begin{array}{c}\text { Local do Evento } \\
\text { (Área Biológica) }\end{array}$ & Local - Edição \\
\hline SICUSP & 1997 & 5 & Ribeirão Preto & São Paulo \\
\hline SICUSP & 1998 & 6 & Ribeirão Preto & São Paulo \\
\hline SIICUSP & 1999 & 7 & Ribeirão Preto & São Paulo \\
\hline SIICUSP & 2000 & 8 & Ribeirão Preto & São Paulo \\
\hline SIICUSP & 2001 & 9 & Ribeirão Preto & São Paulo \\
\hline SIICUSP & 2002 & 10 & Ribeirão Preto & São Paulo \\
\hline SIICUSP & 2003 & 11 & Ribeirão Preto & São Paulo \\
\hline SIICUSP & 2004 & 12 & Ribeirão Preto & São Paulo \\
\hline
\end{tabular}

Este evento foi inicialmente denominado SICUSP (Simpósio de Iniciação Científica da Universidade de São Paulo) e era destinado à apresentação de trabalhos produzidos pelos graduandos da USP. Ao longo dos anos, ele se estendeu a outras instituições até que, em 1999 recebeu a denominação de Simpósio Internacional de Iniciação Científica da USP (SIICUSP), passando a ter caráter internacional (VILLA et al, 2004).

Para a análise das informações contidas nos resumos dos trabalhos, foi elaborado um instrumento de coleta de dados em forma de fichas para quantificar os elementos a serem considerados: instituição/departamento a qual pertence o autor, agência que financiou o estudo, tipo de estudo e temática abordada.

Nesta etapa foram encontrados obstáculos como: insuficiência de informações e falta de objetividade e clareza de alguns títulos e conteúdos dos resumos dos estudos analisados. Também foram encontradas informações incoerentes quanto às instituições às quais estavam vinculados os autores, o que foi solucionado com o auxílio de sites de busca na Internet.

A proposta de classificação de temas adotada neste estudo foi a mesma empregada em estudo anterior sobre a temática, desenvolvido por PEREIRA et al (1999), para possibilitar comparação posterior. Tal classificação inclui as categorias: ensino; aspectos biológicos, químicos e/ou físicos; aspectos psíquicos e comportamentais; aspectos sócioculturais; revisão de literatura; estudos sobre serviços de saúde e trabalho.

A categoria ensino englobou os trabalhos que abordaram o ensino formal, cursos extracurriculares e programas educativos e de orientação em saúde em instituições de ensino. Nos aspectos biológicos, químicos elou físicos se encontram os estudos ligados ao funcionamento do organismo, a drogas e a aspectos nutricionais. Nos aspectos psíquicos e comportamentais foram incluídos resumos sobre o comportamento e os sentimentos das pessoas em determinadas situações, bem como, sobre medidas de percepção. Os aspectos sócio-culturais englobaram assuntos referentes ao conhecimento, informação, valores e práticas de saúde em camadas específicas da sociedade. Em revisão de literatura, constaram trabalhos que consistiam em levantamentos bibliográficos de áreas específicas do conhecimento. Na categoria serviços de saúde, foram incluídas pesquisas voltadas para a assistência à saúde em geral ou para questões a ela relacionadas, com o foco em instituições concretas. A categoria trabalho reuniu estudos cuja problemática estava associada a características laborais e ao processo saúde-doença em classes de trabalhadores.

A classificação por temática se apresentou como um processo complexo devido à possibilidade de se incluir alguns resumos em mais de uma 
categoria. Nestes casos, buscou-se enquadrar a pesquisa na categoria mais coerente com o objetivo central da mesma.

Quanto aos tipos de pesquisa, os estudos foram categorizados em: não-experimentais, que correspondem a pesquisas descritivas; quaseexperimentais, onde são enquadradas as analíticas; e experimentais, conforme proposto por POLIT \& HUNGLER (2004).

Os resultados foram confrontados com 0 contexto em que são produzidos e foram descritos os fatores que podem ter influenciado no perfil da produção científica analisada. Os trabalhos do período citado foram comparados com os de 1993 a 1997 (PEREIRA et al, 1999) e classificados de acordo com os mesmos critérios.

\section{RESULTADOS E DISCUSSÃO}

No período estudado foram apresentados um total de 950 estudos no SIICUSP, que foram publicados sob a forma de resumos em Anais. O número de estudos encontrados em cada ano está apresentado no Gráfico 1:

Gráfico 1: Distribuição dos resumos da área de enfermagem dos Anais dos Simpósios Internacionais de Iniciação Científica de Universidade de São Paulo (SIICUSP) no período de 1997-2004, de acordo com o ano de realização do evento.

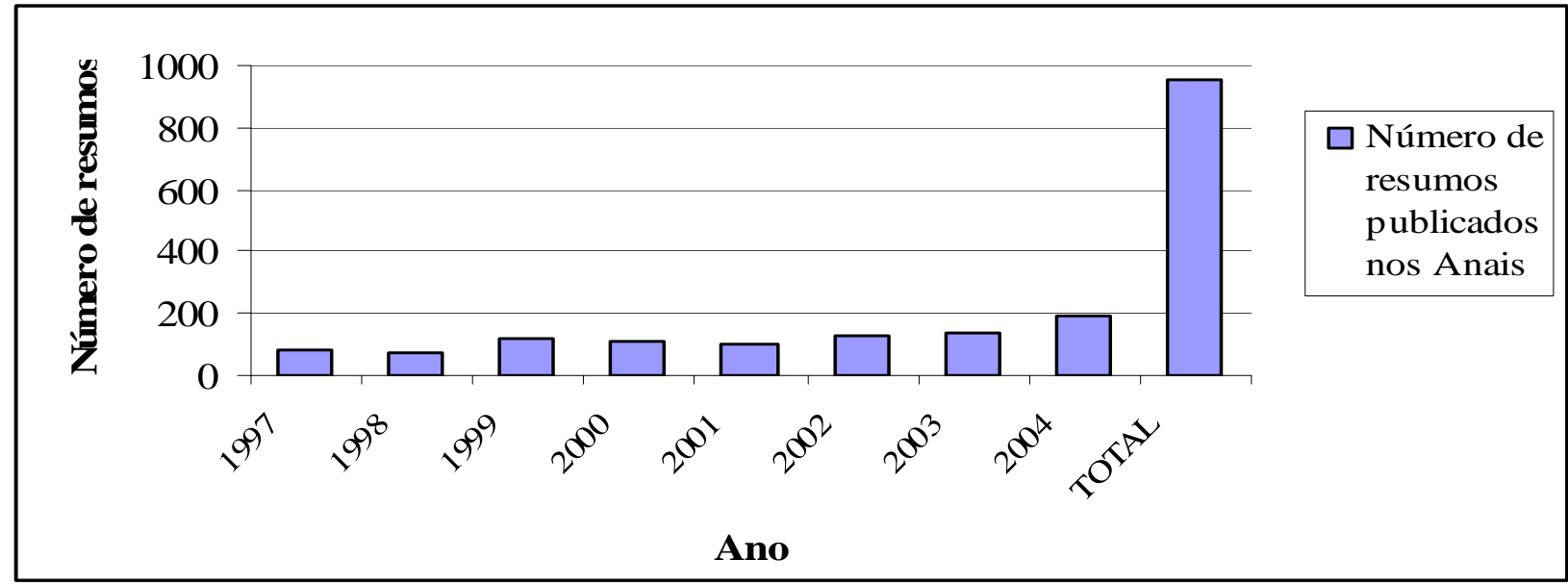

Uma análise do Gráfico 1 revela que houve um aumento significativo no número de trabalhos apresentados nos anos de 2002 a 2004 (458 pesquisas apresentadas) quando comparado aos três primeiros anos em análise neste estudo (276 pesquisas apresentadas). Tal aumento pode ter sofrido a interferência de diversos fatores, entre eles: a abertura para a participação de outras Universidades no evento (VILLA et al, 2004); a divulgação do SIICUSP nessas universidades nos últimos anos; o estímulo fornecido pelos orientadores para que seus orientandos participem do SIICUSP; a exigência por parte de agências de fomento para que o bolsista exponha seus trabalhos. É notável, também, a relação existente entre esse aumento da produtividade e o aumento do fomento concedido no decorrer dos anos (ver Gráfico 4).

Os dados apresentados no Gráfico 2 retratam as temáticas desenvolvidas nos trabalhos científicos. 
Gráfico 2: Classificação dos resumos da área de enfermagem dos Anais dos Simpósios Internacionais de Iniciação Científica de Universidade de São Paulo (SIICUSP) no período de 1997-2004, segundo sua temática.

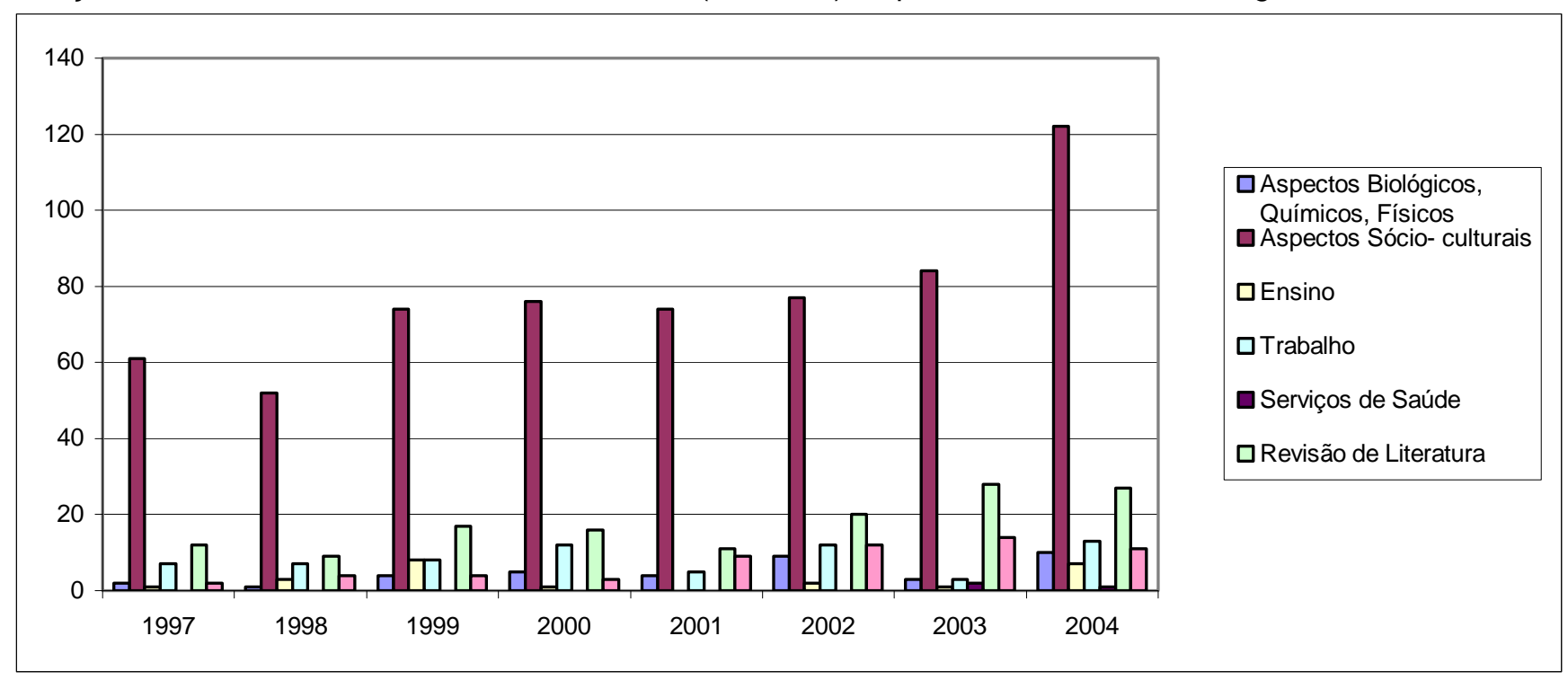

Os aspectos sócio-culturais foram abordados em $65,3 \%$ do total de estudos analisados. Dentre os fatores que possivelmente influenciaram na opção por essa temática estão: a posição da enfermagem, enquanto área do conhecimento, no ponto de convergência entre as ciências humanas e biológicas e o peso relativo de disciplinas das ciências sociais no currículo da graduação em enfermagem. Mostrase, ainda, relevante neste contexto a necessidade de que o enfermeiro tenha uma visão holística, destacando a subjetividade e a singularidade do usuário, bem como a apreensão de termos como vínculo, acolhimento, afetividade e respeito para superar as conseqüências de um ensino fragmentado e reducionista (PINHO et al, 2006).

A segunda categoria de temática mais abordada foi revisão de literatura que, entretanto, apresentou um número menos significativo de pesquisas $(14,7 \%)$. O item serviços de saúde apareceu em apenas $3(0,3 \%)$ dos trabalhos analisados no período. Isso nos alerta para o provável desinteresse dos alunos por um assunto de grande importância para proporcionar ao graduando uma formação adequada.
No estudo de PEREIRA et al (1999), que serve de marco de comparação para a presente investigação, nos ano de 1993 a 1996, os aspectos sócio-culturais também se destacaram entre os temas abordados e o tópico serviços de saúde apareceu em apenas 0,3\% dos resumos.

Evidenciou-se resumos de trabalhos produzidos em anos diferentes que apresentaram conteúdos extremamente parecidos. Isso pode representar um problema relacionado à falta de motivação dos autores, revelando a busca de conveniência ao deixar de cultivar seu potencial para criatividade se distanciando do que deveria ser a atividade de iniciação científica.

Uma parcela dos resumos analisados apresentou falha quanto à explicitação de informações relevantes, tais como: a metodologia adotada, população em estudo, objetivos propostos e considerações finais ou conclusões. Evidenciou-se, ainda, a existência de pesquisas que não apresentavam aprofundamento teórico-metodológico bem como clareza no conteúdo.

O Gráfico 3 expõe as instituições públicas e particulares que participaram do evento apresentando trabalhos no período estudado. 
Gráfico 3: Distribuição do total de pesquisas apresentadas nos Anais dos Simpósios Internacionais de Iniciação Científica da Universidade de São Paulo (SIICUSP) no período de 1997- 2004, segundo as Instituições e/ou departamentos de origem.

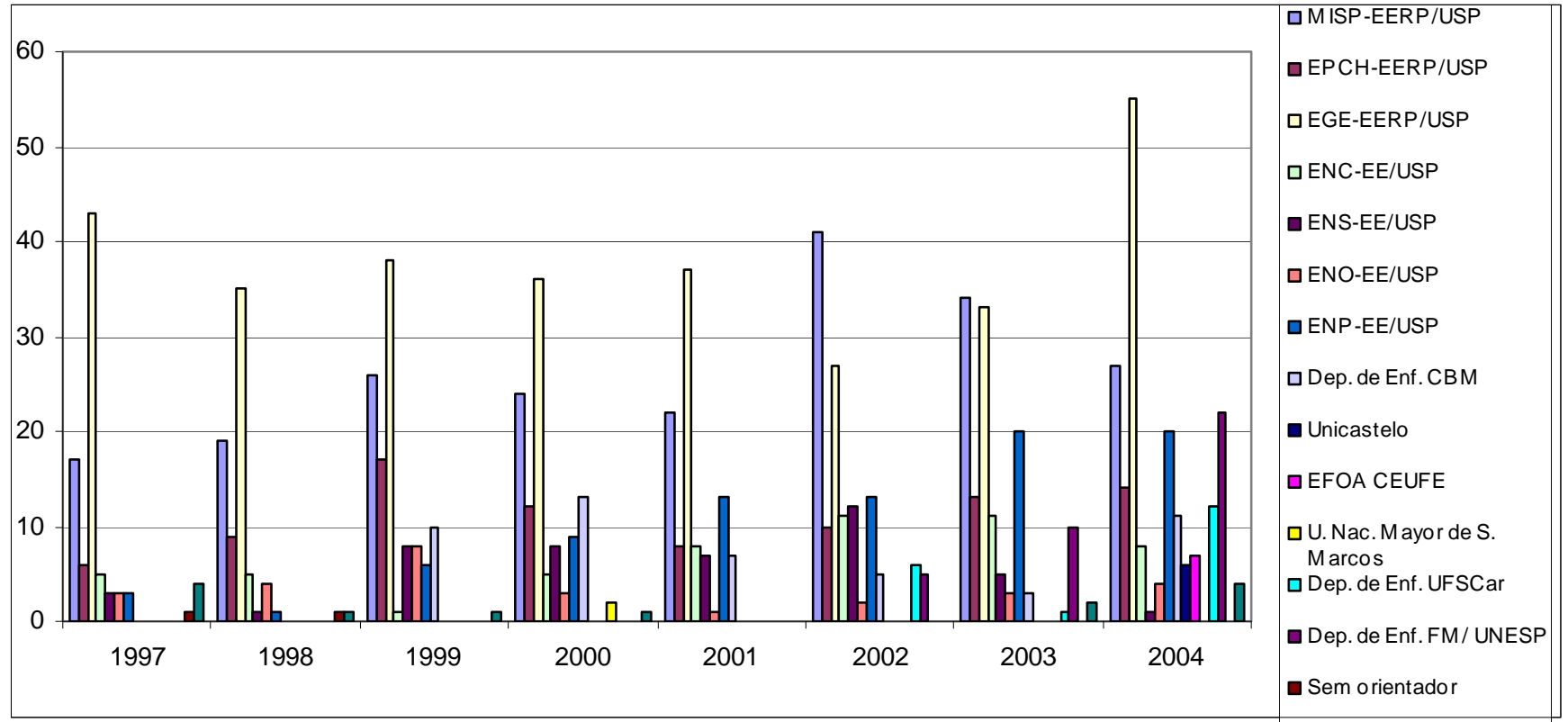

Legenda:

Departamentos da Escola de Enfermagem de Ribeirão Preto da Universidade de São Paulo (EERP/USP): MISP (Enfermagem Materno-

Infantil e Saúde Pública); EPCH (Enfermagem Psiquiátrica e Ciências Humanas); EGE (Enfermagem Geral e Especializada).

Departamentos da Escola de Enfermagem da Universidade de São Paulo (EE/USP): ENC (Enfermagem Médico-Cirúrgica); ENS

(Enfermagem em Saúde Coletiva); ENO (Orientação profissional); ENP (Enfermagem Materno-Infaltil e Psiquiátrica).

Dep. de Enf. CBM: Departamento de Enfermagem do Centro Universitário Barão de Mauá.

Unicastelo: Universidade Camilo Castelo Branco.

EFOA CEUFE: Escola de Farmácia e Odontologia de Alfenas

U. Nac. Mayor de S. Marcos: Universidad Nacional Mayor de San Marcos (Lima- Peru)

Dep. de Enf. UFSCar: Departamento de Enfermagem da Universidade Federal de São Carlos.

Dep. de Enf. FM/UNESP Departamento de Enfermagem da Faculdade de Medicina da Universidade Estadual Paulista.

Vale ressaltar que na categoria "outros" foram incluídas as seguintes instituições e departamentos: Departamento de Patologia e Disciplina de Obstetrícia da Faculdade de Medicina de Ribeirão Preto/USP, Departamento de Saúde Pública da USP, Universidade de Alfenas, Faculdades Metropolitanas Unidas, União das Faculdades da Fundação Hermínio Ometo de Aras, Faculdade de Ciências Administrativas e Contábeis de Lins e Universidade de Taubaté.

No que se refere à origem dos trabalhos analisados nesse estudo (no Gráfico 3), constatou-se que a maioria dos mesmos procede de universidades públicas $(92,6 \%)$, o que revela a importância destas na produção do conhecimento.

Existem características comumente encontradas em universidades públicas que certamente contribuem para a inserção dos alunos nas atividades de pesquisa. Entre elas, estão: cultura organizacional da instituição que tem a pesquisa como missão, a possibilidade de aquisição de uma bolsa de iniciação científica junto às agências de fomento e as exigências por parte da instituição para que os docentes produzam conhecimento científico.

Escolas públicas do Estado de São Paulo contribuíram com $92,5 \%$ da produção analisada. Vale ressaltar que tais escolas concentram menores números de vagas anuais, têm uma quantidade maior de docentes e possuem mais professores atuando em regime de tempo integral quando comparadas com as universidades privadas (DELL'ACQUA \& MIYADAHIRA, 2002). Isto seguramente interfere na disponibilidade do docente para se dedicar à pesquisa e a formação de novos pesquisadores.

A articulação entre a graduação e a pósgraduação, favorecendo o intercâmbio de idéias e experiências; a inclusão de disciplinas relativas ao processo de pesquisar no currículo de graduação; a existência de grupos e núcleos de pesquisa na instituição; o estímulo para a apresentação dos estudos em congressos e simpósios faz parte de um contexto favorável para a adesão e continuidade na atividade de pesquisa por alunos de graduação, contexto este comumente encontrado em Universidades públicas.

A EERP-USP destacou-se pelo número de produções, pois contribuiu com $63,5 \%$ do total de pesquisas, a EE-USP, em segundo lugar quanto ao número de pesquisas, produziu $22,4 \%$ dos trabalhos analisados. Nesse contexto, faz-se importante mencionar que a partir do II Simpósio de Iniciação Científica da USP, em setembro de 2004, foi introduzida a participação de todos os alunos de iniciação científica da Universidade, sendo obrigatória para aqueles ligados ao PIBIC (ALENCASTRE et al, 1996). 
Tem-se, ainda, que em 1988 a EERP-USP foi designada como Centro Colaborador da OMS para o desenvolvimento da pesquisa em enfermagem, o que, certamente, potencializou o senso de responsabilidade e de compromisso para o desenvolvimento da investigação em enfermagem (VILLA et al, 2004).

O espaço garantido pela pós-graduação da EERP/USP por sua produção junto aos pares da comunidade científica (ALMEIDA et al, 2002) é outro fator a ser considerado, pois uma pós-graduação Stricto Sensu bem alicerçada contribui para a consolidação dos grupos e núcleos de pesquisa na unidade, bem como para a definição e consolidação das linhas de pesquisa.

Outro dado encontrado nesta investigação que se distingue do período de 1993 a 1996 (PEREIRA et al, 1999) é a crescente participação de outras universidades brasileiras e a participação de uma universidade peruana no SIICUSP. Tal fato é influenciado pela abertura do evento a outras universidades nacionais e internacionais.

O Gráfico 4 retrata a distribuição do fomento pelas diversas agências em cada ano estudado.

Gráfico 4: Classificação dos resumos da área de enfermagem dos anais dos Simpósios Internacionais de Iniciação Científica de Universidade de São Paulo (SIICUSP) no período de 1997-2004, segundo agência de fomento a pesquisas.

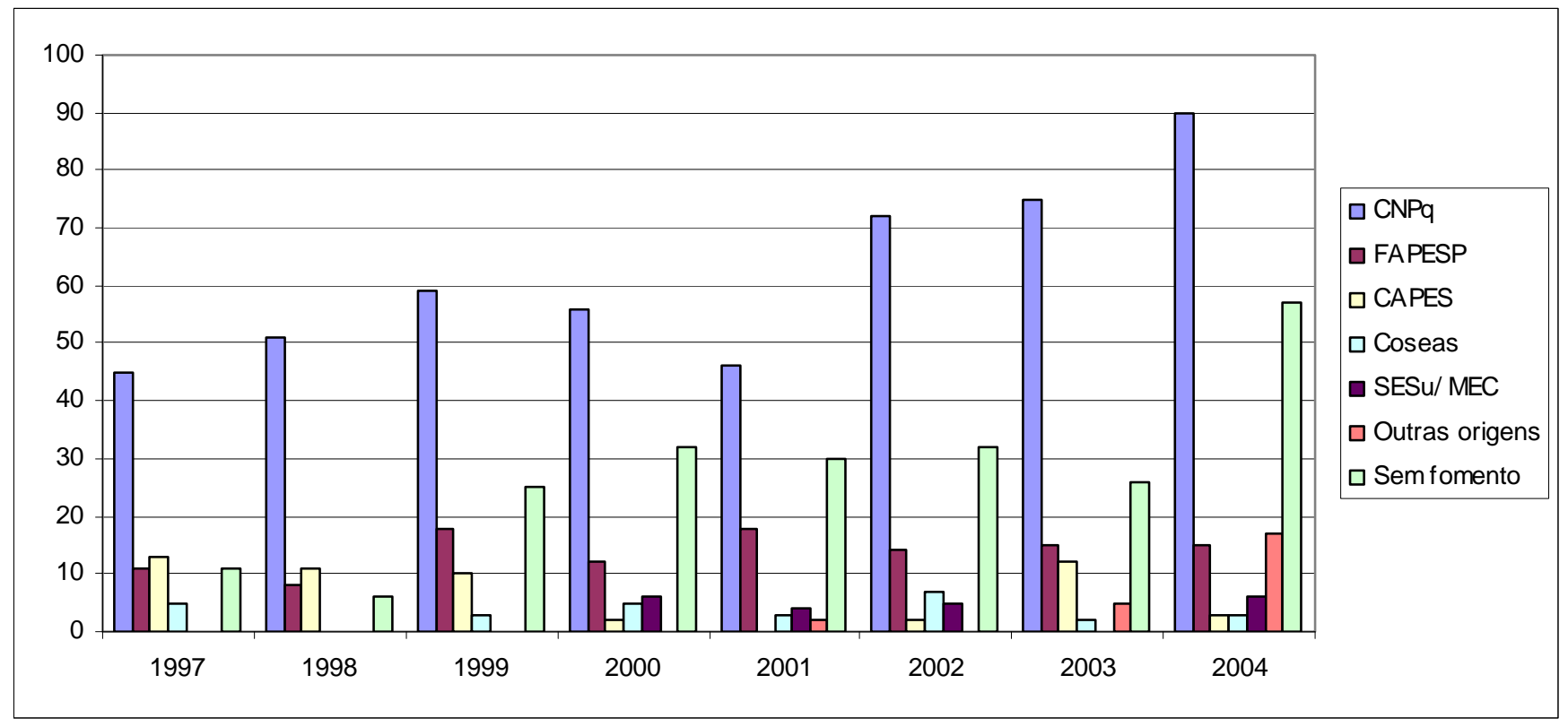

Legenda:

CNPq: Conselho Nacional de Desenvolvimento Científico e Tecnológico.

FAPESP: Fundação da Amparo à Pesquisa do Estado de São Paulo.

CAPES:Coordenação de Aperfeiçoamento de Pessoal de Nível Superior

Coseas: Coordenadoria de Assistência Social

SESu/MEC: Secretaria de Educação Superior/Ministério da Educação

No que se refere à concentração de fomento, $74,4 \%$ dos estudos foram subsidiados por agências ou outras fontes de fomento. Entre tais agências, a que tem financiado um maior número de pesquisas é o CNPq, com financiamento de $52 \%$ dos trabalhos analisados, o que corresponde a $67,6 \%$ de todas as pesquisas que receberam fomento.

A esse respeito, LEITE et al (2001) ao avaliarem os projetos de pesquisa em enfermagem encaminhados ao CNPq, no período de 1998 a 2000, identificaram maior concentração de bolsas concedidas para Região Sudeste onde se encontram, também, a maioria dos pesquisadores e os programas de pós-graduação.

É válido ressaltar que nos locais onde há concentração do fomento, evidenciou-se uma produção maior de pesquisas, o que reflete a importância do apoio das agências para a produção dos trabalhos. $O$ investimento concedido pelas agências de fomento à pesquisa fornece as condições necessárias para a realização das mesmas, pois concede auxílio no custeio da estrutura e dos materiais que viabilizam o desenvolvimento de projetos.

Além disso, o incentivo que as referidas agências concedem por meio de bolsas de pesquisa, como as de iniciação científica, é um estímulo importante para a formação e capacitação de recursos humanos para produção de conhecimento científico.

O Gráfico 5 demonstra a classificação dos trabalhos de acordo com o tipo de pesquisa. 
Gráfico 5: Classificação dos resumos da área de enfermagem dos Anais dos Simpósios Internacionais de Iniciação Científica de Universidade de São Paulo (SIICUSP) no período de 1997-2004, segundo tipo das pesquisas.

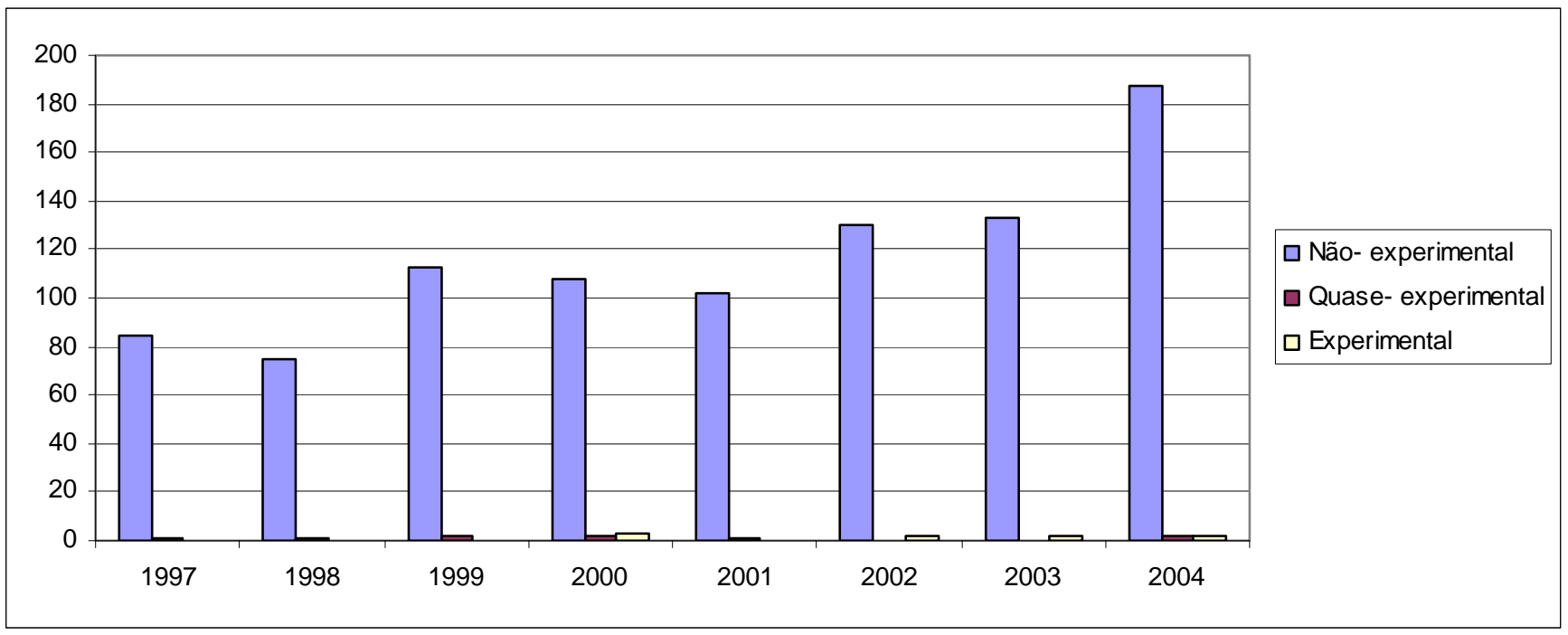

Predominaram as pesquisas nãoexperimentais que corresponderam a $98,1 \%$ do total de estudos apresentados no período em estudo. A pesquisa do tipo não-experimental é caracterizada pela ausência de hipóteses e pelo objetivo de interpretar ou explicar o assunto a ser estudado (POLIT \& HUNGLER, 2004). Este resultado é corroborado por outros estudos que abordam diferentes aspectos da produção científica nacional (BACHION et al, 1992; ALVES, et al. 2004).

Consideramos como possíveis explicações para esse dado a freqüente atuação de professores de outros cursos, principalmente de medicina, ministrando as matérias básicas para os graduandos da enfermagem; a exigência de uma alta produtividade em pesquisa, que é avaliada em termos quantitativos e a falta de capacitação e disponibilidade de tempo dos graduandos para a realização de outros tipos de pesquisas, que requerem maior rigor no controle de variáveis capazes de invalidar os resultados. Tal achado pode estar relacionado, ainda, ao objeto de estudo da enfermagem que demanda, deste profissional, conhecimentos diversos para a abordagem do homem em suas várias dimensões, como por exemplo, biológica, política, social, filosófica, religiosa (COLLET et al, 2000). Muitas dessas dimensões requerem o uso de pesquisas não-experimentais para sua investigação.

Outro aspecto que pode ser considerado como relevante para o predomínio da pesquisa nãoexperimental na enfermagem consiste no processo de formação de pesquisadores enfermeiros brasileiros que, na sua maioria, se doutoram numa perspectiva que não privilegia a pesquisa básica nem a experimental. No entanto, hoje se destaca na formação destes pesquisadores as tendências da pesquisa baseada em evidência, por exemplo, entre outras. A esse respeito, DOMENICO \& IDE (2003) assinalam a prática baseada em evidências na enfermagem como uma prática inovadora que tem se desenvolvido, principalmente, por meio da pesquisa básica.

Entretanto, a pesquisa experimental e quaseexperimental também é necessária para a construção de um corpo de conhecimentos que sirva como guia no estabelecimento de ações que possam conduzir com segurança o cuidado prestado pela enfermagem, garantindo o espaço do enfermeiro na equipe de saúde (ALVES et al, 2004).

A análise comparativa entre os estudos apresentados no SICUSP (atual SIICUSP) no período de 1993 a 1996 e os trabalhos referentes aos anos de 1997 a 2004 demonstrou que há características comuns aos dois períodos: um número expressivo de trabalhos voltados para o estudo de aspectos sócioculturais, um índice elevado de pesquisas de cunho descritivo (que equivale a pesquisa nãoexperimental), além do destaque da EERP-USP na formação de maior número de pesquisadores em nível de iniciação científica.

\section{CONSIDERAÇÕES FINAIS}

Tendo em vista os objetivos delineados para a realização do presente estudo de analisar a produção científica da graduação em enfermagem apresentadas no SIICUSP nos anos de 1997 a 2004, descrever fatores que podem influenciar no perfil dessa produção e estabelecer uma comparação com os trabalhos referentes ao período de 1993 a 1996 (PEREIRA et al, 1999), apresentados no referido evento, destaca-se que sua análise revelou predomínio: de aspectos sócio-culturais entre as temáticas abordadas; das universidades públicas na produção de conhecimento científico e de estudos do tipo não-experimental. A maioria das pesquisas são financiadas por agências de fomento. 
Nos últimos anos, não ocorreram grandes alterações na produção científica da graduação em enfermagem no que se refere aos tipos de pesquisa, temáticas abordadas, agências fomentadoras e instituições/departamentos de origem. Estes dados permitem apreender que: a opção pelo tipo de pesquisa entre os alunos de graduação parece refletir o que ocorre na pós- graduação Stricto Sensu e na enfermagem como um todo, que desenvolve a maioria dos seus trabalhos com a metodologia da pesquisa não-experimental. A procedência das pesquisas é um achado que está profundamente atrelado aos incentivos de agências de fomento a pesquisas. Por outro lado, a preponderância das temáticas apontam para aspectos para os quais a enfermagem comumente está voltada em sua atuação profissional e que, por vezes, é o diferencial da mesma, pois a abordagem de aspectos sócioculturais reflete o interesse em conhecer o indivíduo ao qual é destinado o cuidado bem como o contexto no qual ele se insere.

Considera-se importante atentar para que a iniciação científica não se desvincule das necessidades da sociedade atual e dos assuntos que motivam o aluno, cuidando para que 0 questionamento, o inconformismo, capacidade de reflexão e análise crítica sejam gerados e mantidos entre os graduandos, para que estes descubram o verdadeiro sentido e a responsabilidade de produzir conhecimento científico.

O aluno pode assim observar, analisar, criticar e buscar informações sobre os problemas da realidade, a fim de escolher alternativas e apresentar e executar soluções embasadas na análise. Essa é a finalidade do ensino do método científico nos cursos superiores (CASSIANI \& RODRIGUES, 1998).

A formação de pesquisadores deve ter início na graduação, pois é nessa etapa também que devese apresentar e valorizar a cultura do consumo e produção de pesquisa. Somente com esforços para capacitação de quem se inicia no caminho da pesquisa será possível garantir o crescimento, a qualidade, a continuidade e a valorização da produção de conhecimento na área.

Para isso, julga-se necessárias análises constantes dos rumos da produção científica para identificar progressos, lacunas e mudanças, possibilitando aos pesquisadores repensar de forma crítica seu atuar como produtores de conhecimento.

\section{REFERÊNCIAS BIBLIOGRÁFICAS}

ALENCASTRE, M.B. et al. Programa institucional de bolsas de iniciação científica: experiência da Escola de Enfermagem de Ribeirão Preto da Universidade de São Paulo. Revista Latino-americana de enfermagem, v.4, n.2, 1996.

ALMEIDA, M.C.P. et al. A pós-graduação na escola de enfermagem de Ribeirão Preto-USP: evolução histórica e sua contribuição para o desenvolvimento da enfermagem. Revista Latino-americana de enfermagem, v.10, n.3, p.276-87, 2002.

ALVES, L.M.M.; NOGUEIRA, M.S.; GODOY, S. et al. Pesquisa básica na enfermagem. Revista Latinoamericana de enfermagem, v.12, n.1, p.122-7, 2004. BACHION, M.M. et al. Um estudo sobre a pósgraduação em enfermagem no Brasil, no período de 1962-1991. In: Encontro Internacional "Pesquisa em Enfermagem: Uma questão de saúde". Anais. São Paulo: EE-USP, 1992. p.140.

BAGGIO, M.A. O Significado de cuidado para profissionais da equipe de enfermagem. Revista Eletrônica de Enfermagem, v.8, n.1, p.09-16. [on-line] 2006. Disponível em: http://www.fen.ufg.br/revista/revista8 1/original 01.ht m. [Acesso em 26 de jul. 2006].

CAMACHO A.L.F.; SANTO F.H.E. Refletindo sobre o cuidar e o ensinar na enfermagem. Revista Latinoamericana de enfermagem, v.9, n.1, p.13-7, 2001. CASSIANI S.H.B.; RODRIGUES L.P. O ensino da metodologia científica em oito escolas de enfermagem da Região Sudeste. Revista Latinoamericana de enfermagem, v.6, n.2, p.73-81, 1998 COLLET N.; SCHIEIDER J.F.; CORREA A.K. A pesquisa em enfermagem: avanços e desafios. Revista Latino-americana de enfermagem, v.53, n.1, p.75-80, 2000.

DELL' ACQUA M.C.Q.; MIYADAHIRA A.M.K. Ensino do Processo de Enfermagem nas escolas de graduação em enfermagem no estado de São Paulo. Revista Latino-americana de enfermagem, v.10, n.2, p.185-91, 2002.

DEMO, P. Educar pela pesquisa. Campinas (SP): Ed. Autores associados; 1996.

DOMENICO, E.B.L.D.; IDE, C.A.C. Enfermagem baseada em evidências. Revista Latino-americana de enfermagem, v.11, n.1, p 115-8, 2003.

FERNANDES J.D et al. Articulações da pósgraduação com a graduação no ensino de enfermagem. Revista Baiana de Enfermagem, v.13, n.1/2, p.39-44, 2000.

GUTIÉRREZ M.G.R et al. Os múltiplos problemas pesquisados e a pesquisar na enfermagem. Revista Brasileira de Enfermagem, v.55, n.5, p.535-41, 2002. LEITE, J.L.; TREZZA, M.C.S.F; SANTOS, R.M; MENDES, I.A.C.; FELLI, V.E.A. Os projetos de pesquisa de enfermagem no CNPq: seu percurso, suas temáticas, suas aderências: 1998/2000. Revista Brasileira de Enfermagem, v.54, n.1, p.81-97, 2001.

MENDES, I.A.C. Pesquisa em Enfermagem: Impacto na prática. São Paulo (SP): Editora da Universidade de São Paulo; 1991.

NASCIMENTO, M.A.L.; FIGUEIREDO, N.M.A.; PORTO, F.R. Estímulo à pesquisa em enfermagem: criando pontes entre a graduação e a pós-graduação. Revista de Enferm. UERJ, v.10, n. 1, p. 25-28,2002. PEREIRA et al. A Iniciação científica na graduação em enfermagem na Universidade de São Paulo (1993 a 1996): análise crítica. Revista Latino-americana de enfermagem, v.7, n.3, p.77-86, 1999. 
PINHO, I. C. et al. As percepções do enfermeiro acerca da assistência. Revista Eletrônica de Enfermagem, v.08, n.01, p 42-51, v.08, n.01, p 4251. [on-line] 2006. Disponível em: http://www.fen.ufg.br/revista/revista8 1/original 05.ht $\mathrm{m}$. [Acesso em 26 de jul. 2006].POLIT, D.; HUNGLER, B. Fundamentos de pesquisa em enfermagem: métodos, avaliação e utilização. $5^{a}$ ed. Porto Alegre (RS): Artmed; 2004.

SIICUSP - Simpósio Internacional de Iniciação científica da Universidade de São Paulo, 8, 2000, São Paulo. Programa e resumos. São Paulo, 2000.

SIICUSP - Simpósio Internacional de Iniciação científica da Universidade de São Paulo, 11, 2003, São Paulo. Programa e resumos. São Paulo, 2003.

SOUSA, V.D.; BARROS, A.L.B.L. O ensino do exame físico em escolas de graduação em enfermagem do município de São Paulo. Revista Latino-americana de enfermagem, v.6, n.3, p.11-22, 1998.

VILLA, T.C.S. et al. A Comissão de Pesquisa da Escola de Enfermagem de Ribeirão Preto da Universidade de São Paulo: objetivo e atuação (1989 - 2003). Revista Latino-americana de enfermagem, v.12, n.5, p.828-33, 2004. 